\title{
Greater Biosynthetic Liver Dysfunction in Primary Sclerosing Cholangitis Suggests Co-existent or Impending Cholangiocarcinoma
}

\author{
Panagiotis Trilianos ${ }^{1,2 *}$, Abhishek Agnihotri ${ }^{1}$, Enver Ucbilek $^{1}$ and Ahmet Gurakar ${ }^{1}$ \\ ${ }^{1}$ Department of Gastroenterology \& Hepatology, Section of Transplant Hepatology, The Johns Hopkins School of Medicine, \\ Baltimore MD, USA; ${ }^{2}$ Department of Medicine, MetroWest Medical Center, Framingham, MA, USA
}

\begin{abstract}
Background and Aim: Patients with primary sclerosing cholangitis (PSC) who develop cholangiocarcinoma (CCA) have a median survival of less than 6 months. In half of cases, PSC and CCA will be diagnosed either concurrently or within a year of one another. The aim of the present study is to demonstrate that the degree of biochemical liver dysfunction is associated with concomitant or impending CCA. Methods: We did a chart review of patients diagnosed with PSC and CCA up to 18 months from presentation ("CCA" group) as well as patients with PSC that underwent transplantation with no sign of CCA in their explanted liver ("nCCA" group). Along with demographic data and follow-up length, we recorded their presenting liver function tests, including alanine and aspartate aminotransferases (ALT, AST), total bilirubin (TBil), alkaline phosphatase (ALP), international normalization ratio (INR), and serum Ca 19-9 levels. Differences between mean values of the two groups were analyzed with a student's t-test. Results: Twenty-four patients were included. The "CCA" group consisted of eight patients, and the "non-CCA" group had 16 patients. There was no significant difference between the two groups in their presenting values of ALT, ALP, or serum Ca 19-9. However, the "CCA" group had significantly higher levels of AST, TBil, and INR. Conclusion: Patients with PSC and concurrent or impending CCA appear to exhibit significantly greater biochemical liver dysfunction than those who do not develop CCA. Therefore, newly-diagnosed PSC patients presenting with these findings may warrant more rigorous evaluation.

(C) 2016 The Second Affiliated Hospital of Chongqing Medical University. Published by XIA \& HE Publishing Inc. All rights reserved.
\end{abstract}

Keywords: Cholestatic disease; Malignancy; Liver transplantation; Liver function tests.

Abbreviations: ALP, alkaline phosphatase; ALT, alanine aminotransferase; AST, aspartate aminotransferase; CCA, cholangiocarcinoma; IBD, inflammatory bowel disease; IL-6, interleukin 6; INR, international normalized ratio; IRB, Institutional Board Review; LRG1, leucine-rich a2 glucoprotein 1; NKG2D, natural killer group 2 member D; OLT, orthotopic liver transplantation; PSC, primary sclerosing cholangitis; TBil, total bilirubin.

Received: 6 December 2016; Revised: 29 January 2016; Accepted: 20 January 2016

DOI: $10.14218 / \mathrm{JCTH} .2015 .00048$

*Correspondence to: Panagiotis Trilianos, Dept. of Medicine, MetroWest Medical Center, 115 Lincoln Str., $2^{\text {nd }}$ Floor, Framingham, MA 01701, USA. Tel: 508-5962856, Fax: 508-872-4794, E-mail: Panagiotis.Trilianos@mwmc.com

\section{Introduction}

Cholangiocarcinoma (CCA) is a devastating complication of primary sclerosing cholangitis (PSC) that often results in removal from the liver transplant list and death. In half of cases the malignancy is found within a year of PSC diagnosis. ${ }^{1}$ Early detection of CCA may lead select PSC patients to be included in specialized protocols where chemoradiation precedes and may follow orthotopic liver transplantation (OLT). These protocols have been modestly successful and are reportedly available in about a third of liver transplant centers across the United States. ${ }^{2}$ This emerging potential for cure highlights the importance of identifying risk factors for or warning signs of concurrent or impending CCA in this patient population.

The aim of this study is to examine the association between increased biochemical liver dysfunction, as evidenced by bilirubinemia and coagulopathy, and the presence of synchronous or subsequently developing CCA in patients with PSC.

\section{Methods}

Institutional Board Review (IRB) approval was sought and secured prior to the initiation of the project. We did a chart review of all patients diagnosed and treated for PSC at the outpatient Hepatology clinic of The Johns Hopkins Hospital from 1998 to 2012. Primary end points included diagnosis of CCA or OLT with no evidence of malignancy in the explanted liver. First clinic visit was designated as "Day 0" and age, gender, race, alcohol and tobacco consumption, and coexisting diagnosis of inflammatory bowel disease (IBD) were recorded for each patient. Patients with known concurrent viral hepatitis were excluded from the study, as were those with documented biliary instrumentation prior to or fever and leukocytosis on Day 0 . Labs obtained on Day 0 included aspartate and alanine aminotransferases (AST, ALT), total bilirubin (TBil), alkaline phosphatase (ALP), international normalized ratio (INR), and serum Ca 19-9 antigen levels. Patients with incomplete data sets were eliminated. Patients that developed CCA beyond 18 months of follow-up were excluded from the study. Included in the control group were only those patients that underwent OLT with no sign of CCA in the explanted liver, the rationale being that explant pathology examination would exclude underlying, subclinical CCA in a PSC patient with reasonable confidence. The final study population consisted of patients who developed CCA within 
Trilianos P. et al: Transaminitis, Coagulopathy and Cholangiocarcinoma

18 months of Day 0 ("CCA group") and the control group ("nCCA group"). Mean lab values were calculated for each group and student's t-test was implemented to determine the statistical significance of the differences between means. Chi square test was utilized to detect association of smoking, alcohol, and presence of IBD with eventual development of CCA. A derived $p<0.05$ was considered statistically significant. Software used for statistical analysis was STATA (by StataCorp LP, College Station, TX, USA).

\section{Results}

The records of 43 patients with PSC were retrieved from our electronic database. Sixteen patients were diagnosed with CCA at some point during their follow-up; half of these patients (8) developed CCA within 18 months of Day 0, while the other half developed malignancy at a later time in their course, thus excluding them from the study. Twentyseven patients remained CCA-free as of their last clinic visit, and 21 of them underwent OLT with no evidence of CCA in their explant pathology, over a mean period of 52.9 months. Five of these 21 patients were excluded due to missing laboratory values on Day 0 . Patients that met all our criteria formed a final sample size of 24 (eight in the "CCA" group, 16 in the "nCCA" group). Demographic characteristics included predominance of male sex $(n=6$ in the "CCA", $n=13$ in the "nCCA" group) and Caucasian race ( $\mathrm{n}=7$ in the "CCA", $\mathrm{n}=24$ in the "nCCA" group). Patients in the "CCA" group were of significantly older age ( 48 vs. 36 years, $p=0.017$ ) than those in the "nCCA" group. Tobacco and alcohol use were not quantified for each patient, but their presence was identical in the two groups $(37.5 \%$ and $25 \%$ for smoking and alcohol, respectively). Mean follow-up from Day 0 to CCA detection was 7 months for the "CCA" group; mean follow-up until the time of OLT for the "nCCA" group was 60 months $(p=0.000)$. Prevalence of IBD (irrespective of subtype) was documented in five out of eight $(62.5 \%)$ patients in the "CCA" group and in 12 out of $16(75 \%)$ patients in the "nCCA" group; this difference was not significant $(p=0.52)$. None of the patients of the "CCA" group experienced variceal bleeding, while four of the "nCCA" group had at least one ( $0 \%$ vs $25 \%, p=0.121)$. In terms of liver biochemistry, patients of the "CCA" group on Day 0 were found to have significantly more elevated levels of AST (283.7 vs. 87.7, U/L, $p=0.034$, Fig. 1.), TBil (8.0 vs. 3.2, $\mathrm{mg} / \mathrm{dl}, p=0.042$, Fig. 2 ), and INR (1.17 vs. $1.01, p=0.026$ ) than the "nCCA" group. On the other hand, levels of ALT (185.5 vs. $105.6, \mathrm{U} / \mathrm{L}, p=0.104$ ), ALP (334.0 vs. 491.3 , $\mathrm{U} / \mathrm{L}, p=0.136$ ) or serum Ca $19-9$ (347.0 vs. $116.6, \mathrm{U} / \mathrm{ml}$, $p=0.126)$ did not differ significantly between the two groups. Table 1 features all the demographic and biochemical characteristics for both groups.

\section{Discussion}

The incidence of CCA in PSC has been estimated to be up to $1.5 \%$ per year. ${ }^{3}$ Identifying PSC patients susceptible to developing CCA would allow for implementation of timely interventions. To that end, serum and bile markers for CCA have been sought at the molecular level. An increase in the frequency of Tp53, bcl-2, k-ras, and p16-INK4a mutations in CCA tissue as well as in the bile of PSC patients with the malignancy have been demonstrated, ${ }^{4-6}$ but mutations such as these characterize the tumorigenesis in a variety of human cancers. Melum et al. ${ }^{7}$ found single nuclear polymorphisms in the

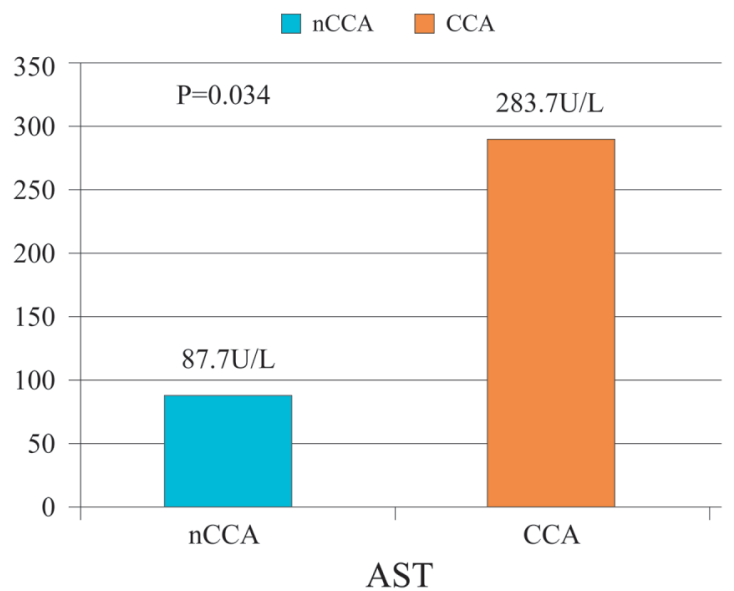

Fig. 1 Aspartate aminotransferase (AST) levels in "nCCA" and "CCA" groups.

natural killer group 2 member D (NKG2D) cell receptor gene associated with an increased risk for CCA in PSC; the authors noted, however, that the detection of the predisposing alleles as a means to isolate the high-risk patients would yield too many false positives. Elevated serum levels of leucine-rich a2 glucoprotein 1 (LRG1), alone or in combination with interleukin 6 (IL-6) and Ca 19-9, were shown to significantly aid in the discrimination between malignant and benign biliary strictures. $^{8}$ Bile $^{9}$ and urine ${ }^{10}$ proteomic analyses and most recently the detection of extracellular vesicles in the bile and specific arrays of microRNAs within those vesicles ${ }^{11}$ show promise in distinguishing underlying malignancy from a PSC flare.

The degree of biochemical deterioration, serum Ca 19-9 levels, presence and duration of IBD, alcohol consumption, and smoking have all been suggested as risk factors for the development of CCA in this population, along with a history of colonic dysplasia, cancer, proctocolectomy, and variceal bleeding.

In the largest study of its kind, ${ }^{1}$ the European Study Group of Primary Sclerosing Cholangitis retrospectively examined the records of 394 consecutive PSC patients, 48 of whom $(12.2 \%)$ developed CCA, half of them within a year of PSC diagnosis. The latter subgroup was found to contribute to

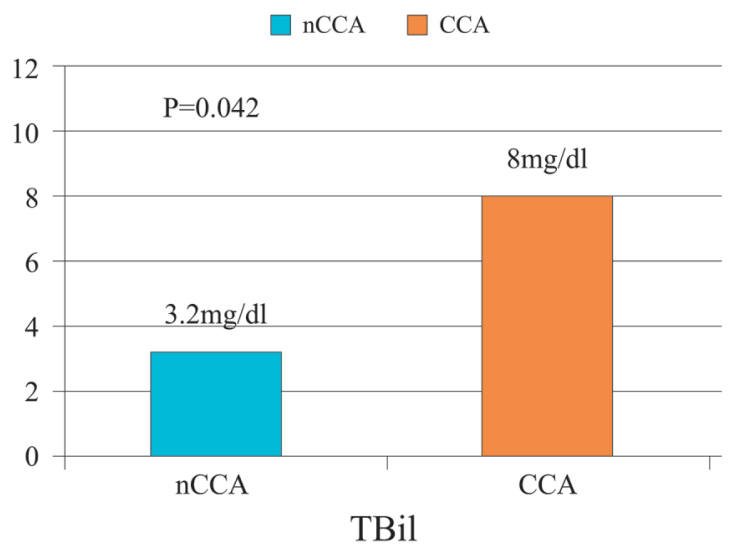

Fig. 2 Mean total bilirubin in "nCCA" and "CCA" groups. 
Trilianos P. et al: Transaminitis, Coagulopathy and Cholangiocarcinoma

Table 1. Characteristics of "CCA" and "nCCA" groups based on demographic and biochemical data

\begin{tabular}{llll}
\hline Variable & No CCA & CCA & $p$ value \\
\hline Age (mean, years) & 36 & 48 & $\mathbf{0 . 0 1 7}$ \\
Race, White & 16 & 6 & 0.148 \\
Sex, Male & 11 & 6.7 & 0.750 \\
Follow-up (mean, months) & 60 & 5 & $\mathbf{0 . 0 0 0}$ \\
IBD & 12 & 1.17 & 0.525 \\
INR @ Day 0 (mean) & 1.01 & 185.5 & 0.026 \\
ALT @ Day 0 (mean, U/L) & 105.6 & 283.7 & 0.104 \\
AST @ Day 0 (mean, U/L) & 87.7 & 8.0 & $\mathbf{0 . 0 3 4}$ \\
TBil @ Day 0 (mean, mg/dl) & 3.2 & 347 & $\mathbf{0 . 0 4 2}$ \\
CA 19-9 @ Day 0 (mean, IU/ml) & 116.6 & 334 & $\mathbf{0 . 1 2 6}$ \\
ALP @ Day 0 (mean, U/L) & 491.3 & $3(37.5 \%)$ & 0.136 \\
Alcohol (no, percentage) & $6(37.5 \%)$ & $2(25 \%)$ \\
Tobacco (no, percentage) & $4(25 \%)$ & - \\
\hline
\end{tabular}

ALP, alkaline phosphatase; ALT, alanine aminotransferase; AST, aspartate aminotransferase; IBD, irritable bowel syndrome; INR, international normalized ratio.

significantly higher levels of serum bilirubin and coagulopathy compared to the majority of PSC patients that did not develop CCA. In an older, but almost equally sizeable Swedish study of 305 PSC patients, ${ }^{12}$ serum bilirubin at diagnosis of PSC was significantly higher in those who later developed CCA than those who did not. Thus, the available data from highpowered studies confirm our own finding of significantly higher bilirubin levels in the "CCA" group. The aminotransferase levels, however, did not significantly differ between CCA and nonCCA PSC patients in those studies, which is in contrast to our finding of significantly higher AST (but not ALT) levels in the "CCA" group. A very recent study from the University of Hiroshima ${ }^{13}$ examining the biochemical and cholangiographic characteristics of CCA in PSC and featuring an almost equal sample size $(n=23)$ corroborated our findings of both significantly higher bilirubinemia and AST transaminitis in CCA patients.

Elevated Ca 19-9 certainly raises suspicion of CCA and often triggers further evaluation with non-invasive (ultrasound, magnetic resonance cholangiopancreatography) and invasive (endoscopic retrograde cholangiopancreatography with biliary brushings, cholangioscopy) modalities. However, it can also occur in acute/recurrent bacterial cholangitis, benign stricturing of the biliary tree, or for other reasons unknown. Some authors consider serum Ca 19-9 a screening tool that is in itself unreliable and only to be used in combination with imaging and/or cytology. ${ }^{14}$ Sinakos et al. $^{15}$ showed that patients subsequently proven to have benign strictures presented with initial serum Ca 19-9 values of up to $2000 \mathrm{U} / \mathrm{mL}$ (although not exceeding that), and more than a third of all PSC patients with significantly elevated Ca 19-9 did not in fact have CCA. Serum Ca 19-9 levels were found to be significantly elevated in the "CCA" group compared to the controls in the population studied by Ishii et al. ${ }^{13}(47 \mathrm{U} / \mathrm{mL}$ vs $7 \mathrm{U} / \mathrm{mL}, p=0.017)$, whereas in our study, the difference in marker levels between the two groups, albeit marked, did not attain statistical significance (347 U/mL in the "CCA" vs $116.6 \mathrm{U} / \mathrm{mL}$ in the "nCCA" group, $p=0.126)$.
The prevalence of IBD did not differ significantly between PSC patients who did and did not develop CCA in our study or the study by Boberg et al. ${ }^{1}$ The latter, however, did demonstrate that the duration of IBD for the CCA group was significantly longer. An earlier study by Bergquist et al.,16 albeit one of more limited power, showed no association between duration of IBD and the development of CCA but significant frequency of smoking in the PSC/CCA group. Chalasani et al. ${ }^{17}$ also found no association between duration of IBD and CCA but none between smoking and CCA either, implicating alcohol consumption instead. In a multivariate analysis by the Mayo Clinic, ${ }^{3}$ only history of variceal bleeding proved to be a significant risk factor for the development of CCA in PSC patients. In contrast, our population exhibited identical prevalence of tobacco and alcohol use in the two groups. Small size, the comparatively very short follow-up time until the primary end point (CCA), and the poor group survival rates may explain the absence of episodes of variceal bleeding among our "CCA" patients.

The most significant limitation of the present study is the relatively small sample size; which may have contributed to the lack of statistical significance of the difference in mean serum Ca 19-9 between the two groups and the lack of clinically meaningful, albeit statistically significant, difference in coagulopathy, as evidenced by the two groups' mean INRs. A study with a greater sample size may demonstrate a wider gap, thereby linking more strongly biochemical dysfunction and cholangiocarcinoma.

\section{Conclusion}

In summation, we have demonstrated and confirmed the finding of prior large-scale retrospective studies, which found that PSC patients with co-existing or impending CCA showed greater biochemical liver dysfunction, as demonstrated by significantly more increased total bilirubin and INR levels compared to those who do not develop the malignancy. Levels of AST also appeared to be more elevated in patients who develop CCA, possibly due to a greater degree of cholestasismediated liver injury. Amidst the current effort to identify 
sensitive, specific, accurate, and practical markers to detect CCA in this challenging population and until such markers are found and become widely available, we recommend that PSC patients who present with significant bilirubinemia and coagulopathy are intensely evaluated with all available means to exclude the presence of CCA.

\section{Conflict of interest}

None

\section{Author contributions}

Data collection (PT, AA), data analysis and manuscript drafting (PT), manuscript revision (EU, AG).

\section{References}

[1] Boberg KM, Bergquist A, Mitchell S, Pares A, Rosina F, Broomé U, et al. Cholangiocarcinoma in primary sclerosing cholangitis: risk factors and clinical presentation. Scand J Gastroenterol 2002;37:1205-1211. doi: 10. 1080/003655202760373434.

[2] Trilianos P, Selaru F, Li Z, Gurakar A. Trends in pre-liver transplant screening for cholangiocarcinoma among patients with primary sclerosing cholangitis. Digestion 2014;89:165-173 doi: 10.1159/000357445.

[3] Burak K, Angulo P, Pasha TM, Egan K, Petz J, Lindor KD. Incidence and risk factors for cholangiocarcinoma in primary sclerosing cholangitis. Am J Gastroenterol 2004;99:523-526. doi: 10.1111/j.1572-0241.2004.04067.x.

[4] Ahrendt SA, Rashid A, Chow JT, Eisenberger CF, Pitt HA, Sidransky D. p53 Overexpression and K-ras gene mutations in primary sclerosing cholangitis-associated biliary tract cancer. J Hepatobiliary Pancreat Surg 2000;7: 426-431 doi: 10.1007/s005340070039.

[5] Boberg KM, Schrumpf E, Bergquist A, Broomé U, Pares A, Remotti $H$, et al. Cholangiocarcinoma in primary sclerosing cholangitis: K-ras mutations and Tp53 dysfunction are implicated in the neoplastic development. J Hepatol 2000;32:374-380. doi: 10.1016/S0168-8278(00)80386-4.

[6] Taniai M, Higuchi H, Burgart LJ, Gores GJ. p16INK4a promoter mutations are frequent in primary sclerosing cholangitis (PSC) and PSC-associated cholangiocarcinoma. Gastroenterology 2002;123:1090-1098. doi: 10 . 1053/gast.2002.36021.

[7] Melum E, Karlsen TH, Schrumpf E, Bergquist A, Thorsby E, Boberg KM, et al. Cholangiocarcinoma in primary sclerosing cholangitis is associated with NKG2D polymorphisms. Hepatology. 2008;47:90-96. doi: 10.1002/hep. 21964.

[8] Sandanayake NS, Sinclair J, Andreola F, Chapman MH, Xue A, Webster G], et al. A combination of serum leucine-rich $\alpha$-2-glycoprotein 1, CA19-9 and interleukin- 6 differentiate biliary tract cancer from benign biliary strictures. $\mathrm{Br}$ J Cancer 2011;105:1370-1378. doi: 10.1038/bjc.2011.376.

[9] Lankisch TO, Metzger ], Negm AA. Bile proteomic profiles differentiate cholangiocarcinoma from primary sclerosing cholangitis and choledocholithiasis. Hepatology 2011;53:875-884. doi: 10.1002/hep.24103.

[10] Metzger J, Negm AA, Plentz RR. Urine proteomic analysis differentiates cholangiocarcinoma from primary sclerosing cholangitis and other benign biliary disorders. Gut 2013;62:122-130. doi: 10.1136/gutjnl-2012-302047.

[11] Li L, Masica D, Ishida M, Tomuleasa C, Umegaki S, Kalloo AN, et al. Human bile contains microRNA-laden extracellular vesicles that can be used for cholangiocarcinoma diagnosis. Hepatology 2014;60:896-907. doi: 10.1002/hep. 27050.

[12] Broomé U, Olsson R, Lööf L, Bodemar G, Hultcrantz R, Danielsson A, et al. Natural history and prognostic factors in 305 Swedish patients with primary sclerosing cholangitis. Gut 1996;38:610-615. doi: 10.1136/gut.38.4.610.

[13] Ishii $Y$, Sasaki T, Serikawa M, Kobayashi K, Kamigaki M, Minami T, et al. Characteristic features of cholangiocarcinoma complicating primary sclerosing cholangitis. Hepatogastroenterology 2014;61:567-573.

[14] Björnsson E., Kilander A, Olsson R. CA 19-9 and CEA are unreliable markers for cholangiocarcinoma in patients with primary sclerosing cholangitis. Liver Int 1999;19:501-508. doi: 10.1111/j.1478-3231.1999.tb00083.x.

[15] Sinakos E, Saenger AK, Keach J, Kim WR, Lindor KD. Many patients with primary sclerosing cholangitis and increased serum levels of carbohydrate antigen 19-9 do not have cholangiocarcinoma. Clin Gastroenterol Hepatol 2011;9:434-439. doi: 10.1016/j.cgh.2011.02.007.

[16] Bergquist A, Persson B, Broomé U. Risk factors and clinical presentation of hepatobiliary carcinoma in patients with primary sclerosing cholangitis: a case-control study. Hepatology 1998;27:311-316. doi: 10.1002/hep. 510270201.

[17] Chalasani N, Baluyut A, Ismail A, Zaman A, Sood G, Ghalib R., et al. Cholangiocarcinoma in patients with primary sclerosing cholangitis: a multicenter case-control study. Hepatology 2000;31:7-11. doi: 10.1002/hep. 510310103. 